\title{
Machining Characteristics, Tribological and Corrosion Behaviour of AA7075 Hybrid Composites
}

\author{
Abhishek Sharma, P.M. Mishra
}

\begin{abstract}
In this paper an attempt has been made to review machining characteristics, tribological and corrosion behaviour of aluminum alloy 7075 hybrid composite utilizing different types of reinforcements. Many investigations revealed that application of various new techniques like friction stir processing, electric discharge machining and abrasive water jet machining are found to be the successful for enhancing the machining characteristics of hybrid composite. It was found in many researches that the introduction of hard ceramic phase with soft reinforcement phase into the matrix improves the tribological characteristics along with other mechanical properties. The corrosion rate was found to be higher for the composite with poor interface between matrix and reinforcement. Also, many researchers reported about the enhancement of corrosion resistance due to heat treatment, employing friction stir processing technique and optimized addition of reinforcement.
\end{abstract}

Keywords: Hybrid metal matrix composite, AA7075, machining characteristics, wear, corrosion.

\section{INTRODUCTION}

Alumnium alloy 7075 is one of the most popular alloys due to its excellent characteristic properties such as very high strength to weight ratio, good ductility, high toughness, low density, high thermal conductivity and good formability. The problem with this alloy is that they possess very poor wear resistance attributed to severe plastic deformation and material removal under different wear conditions. Many investigations reported about the improvement of tribological characteristics of this alloy by the addition of different reinforcement. Therefore, AA7075 metal matrix composite are believed to good replacement for the monolithic alloys. Apart from single reinforced composite, hybrid reinforced composite is gaining more attention due to enhancement of the overall performance of composite by employing multiple reinforcements. The hybrid composite offers improved

Revised Manuscript Received on July 22, 2020.

* Correspondence Author

Abhishek Sharma*, Department of Mechanical Engineering, Maulana Azad National Institute of Technology, Bhopal, M.P, India (462003). Email: abhi131088@gmail.com

P.M. Mishra, Department of Mechanical Engineering, Maulana Azad National Institute of Technology, Bhopal, M.P, India (462003). Email: 2010pinkumishra@gmail.com

(c) The Authors. Published by Blue Eyes Intelligence Engineering and Sciences Publication (BEIESP). This is an open access article under the CC BY-NC-ND license (http://creativecommons.org/licenses/by-nc-nd/4.0/) performance at reduced cost by the use various cheap reinforcements life industrial and agricultural waste materials. The hybrid composite also known for their higher corrosion resistance than the monolithic alloys. There are various methods of production of hybrid composite such as liquid metallurgy and solid-state processing but much less has been discussed about the machining characteristics of these composites. In this review an attempt has been made by author to shed light on the various researches conducted on the machining characteristics, tribological and corrosion behaviour of AA 7075 hybrid composite.

\section{MACHINING CHARACTERISTICS}

Kumar et al. [1] fabricated hybrid composite with Al2O3 and B4C as reinforcements in matrix of AA 7075 using stir casting technique. Further it was subjected to friction stir processing in order to achieve enhanced mechanical properties. It is well known fact that the mechanical properties are largely dependent on microstructural features of any metal matrix composite. Multi pass friction stir processing was employed mainly to modify the microstructure of composite obtained after stir casting. Since casting often results into agglomeration of reinforcement particles which severely affects the desired microstructures. FSP (Friction Stir Processing) is a solid-state processing method which employs frictional heat, stirring mechanism and multiple passes over the surface. They found that travelling speed of tool hardly effect the distribution of particle in the nugget zone. It was evident that at very low travelling speed very good distribution of powder was achieved. High travelling speed causes improper powder distribution because it gets less time for stirring. Wear resistance was found highest for the sample processed with very high coefficient of friction at lowest travelling speed because of proper distribution of B4C particles. Microstructure of sample processed at very low travelling speed revealed that in stir zone there was uniform dispersion of particles. Consequently, the hardness increased with increase in concentration of particles. Mahanta et al. [2] investigated about various sustainable measures while machining AA 7075 hybrid composite reinforced with fly ash and boron carbide by electric discharge technique. Current, voltage, pulse on time and pulse off time were taken as process parameter while sustainable measures considered were surface roughness and power consumption.

Published By:

Blue Eyes Intelligence Engineering \& Sciences Publication

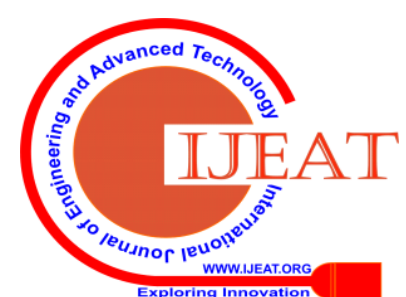




\section{Machining Characteristics, Tribological and Corrosion Behaviour of AA7075 Hybrid Composites}

They also used 3D contour plots for analyzing experimental results and found that for all sustainable measures the main dominating parameters were current and pulse on time. They used response surface methodology for developing full quadratic modeling which exhibited good correlations and also resulted percentage error of 3.76\% for surface roughness and $3.97 \%$ for power consumption. It was evident from surface plot that deeper and wider craters were caused due to high material removal rate and spark energy on increasing current and pulse on time. Kolli et al. [3] reported about the machining characteristics such as material removal rate and surface roughness of AA 7075 hybrid composite reinforced with boron carbide and graphite processed through electric discharge machining. RSM technique with box-behnken design was used for analysis and optimization of process parameter such as voltage, current, pulse on time and pulse off time. ANOVA was utilized for determining optimized level of process parameters. It was found that material removal rate was maximum at voltage $40 \mathrm{~V}$, current 11 Amp, pulse on time $35 \mu$ s, lower levels of pulse off time 15 $\mu$ s whereas surface roughness was minimum at voltage $40 \mathrm{~V}$, current 7 Amp, pulse on time $25 \mu$ s and lower levels of pulse off time $35 \mu \mathrm{s}$. Roy et al. [4] prepared AA7075 hybrid composite reinforced with silicon carbide and carbon black as reinforcements through stir casting. Reinforcements were uniformly distributed throughout the matrix as confirmed by XRD, EDS and FESEM. Composite were then subjected to friction stir processing with considering tool speed, feed rate and tool tilt angle as process parameter. Microstructure revealed that hybrid composite had refined grains which after FSP got modified into ultra-fine equi-axed grains as shown in optical micrographs (Fig.1) [4]. They also reported that UTS of hybrid composite obtained after casting was 2.5 times higher and ductility was also found to be increased than the base alloy. Wear properties were also significantly improved after FSP.
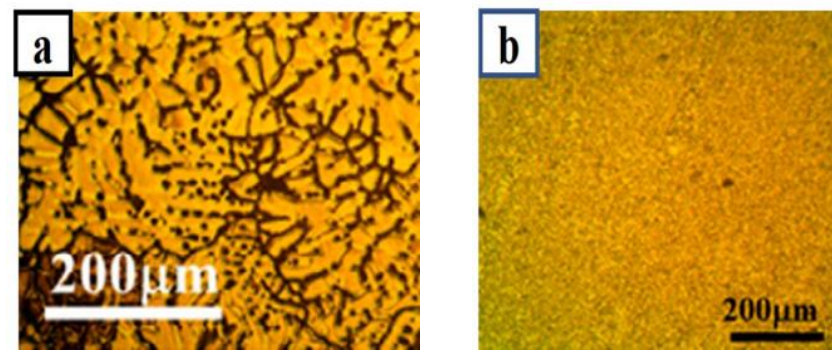

Fig. 1. Optical micrographs of AA7075/SiC/CB a) Without friction stir processing $b$ ) After friction stir processing [4]

Kumar et al. [5] Used $\mathrm{SiC}$ and $\mathrm{TiC}$ as reinforcements with AA7075 as matrix to fabricate hybrid composite by stir casting technique. They utilized EDM for machining the composite and also investigated about the process parameters that influenced the machining. Current, pulse on time and pulse off time were the main process parameters which were considered and their effect on material removal rate and surface roughness was analyzed. Design expert software was used for identifying the optimum value of process parameters. They reported that as concentration of reinforcement particle was increased and surface roughness was decreased the material removal rate was also decreased. Out of three process parameters while using copper tool material removal rate mainly depended on current than pulse on time and was least influenced by pulse off time. While in case of surface roughness it depended largely on pulse on time than the current and here also effect of pulse off time was negligible. Current 13 Amp, pulse on time $50 \mu$ s and pulse off time $8 \mu \mathrm{s}$ were the optimum values for the process parameters of EDM. Sasikumar et al. [6] produced hybrid composite with 7075 alloy as matrix and Titanium Carbide and Boron carbide as reinforcements by two step stir casting technique. Microstructure revealed that both the reinforcements were uniformly distributed except in some cases where slight agglomeration also took place. They investigated about the process parameters of Abrasive water jet machining such as jet traverse speed, water jet pressure and standoff distance and their influence on the kerf characteristics (width, angle and surface roughness). They reported that process parameters had direct influence on kerf characteristics as revealed by ANOVA. They found that on increasing jet traverse speed surface roughness and top kerf width decreased whereas kerf angle increased. For better surface finish and minimum kerf angle pressure of water Jet should be kept high. For achieving low top kerf width and kerf angle and better surface finish less standoff distance should be preferred. Kumar et al. [7] processed hybrid composite with $\mathrm{SiC}$ and Graphite as reinforcement and AA7075 as matrix material. Further they investigated about influence of cutting parameters on roughness while turning the hybrid composite. The cutting parameters taken into account were cutting speed, feed rate and approach angle. They reported that at high cutting speed and low feed rate best surface finish was obtained and approach angle had very less significance. Also, machinability of hybrid composite was improved due to graphite particles which consequently improved the surface finish. They also constructed model with the help of RSM and ANN and found that former was much better than latter because it resulted less error. Lal et al. [8] fabricated hybrid composite using Silicon carbide and alumina particles with AA 7075 using inert gas assisted electromagnetic stir casting technique. Further the composite was subjected to machining using wire electric discharge machining process. They also studied about the effect of process parameters of wire EDM such as discharge duration, discharge current, pulse interval time and wire drum speed on the kerf width. They utilized analysis of variance for determining level of importance and used Taguchi method for optimizing parameters. Among the process parameters discharge current, discharge duration and wire drum speed were found to be the most significant. Kerf width was most influenced by discharge current $47.16 \%$ and then by discharge duration $38.36 \%$, wire drum speed $5.16 \%$ and interaction between first two $5.47 \%$. Kerf width was least affected by pulse interval time.

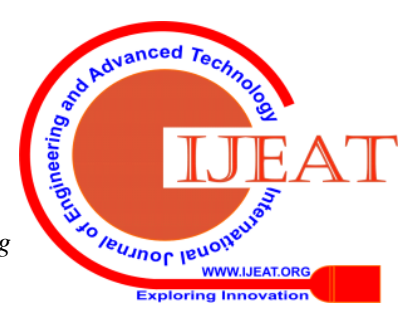




\section{TRIBOLOGICAL BEHAVIOUR}

Y1ldırım et al. [9] used 4\% Ti and 9\% B4C in Al alloy 7075 to prepare hybrid composite via powder metallurgy technique. They observed homogeneous distribution of all the phases in the composite.

They tested the sample for their wear behavior at sliding speed of $1 \mathrm{~ms}-1$, load $30 \mathrm{~N}$ and sliding distance between 500-3000 m. It was found that with increase in sliding distance weight loss was increased. Kumar et al. [10] fabricated hybrid composite with Bagasse ash and graphite as reinforcement by stir casting technique. They studied about their tribological behavior using Taguchi method and found optimal combination of parameters for minimizing the wear and also for minimizing frictional force. Swathi et al. [11] used alumina and graphite as reinforcement and AA7075 as reinforcement for processing hybrid composite through stir casting. Composite was subjected to wear test using Taguchi method. They found that alumina being hard phase strengthened the matrix by resisting the movement of dislocations that consequently enhanced the wear resistance of composite. The film of graphite prevented the breaking of alumina particles. They found that frictional force increased with content of alumina. Basithrahman et al. [12] fabricated AA7075 hybrid composite by stir casting technique in which they used Alumina, Titanium Dioxide and boron carbide particles as reinforcements. Wear tests revealed that with increase in content of reinforcements wear resistance increased. Imran et al. [13] used bagasse ash and graphite particles with AA7075 as matrix for processing hybrid composite. The composite was tested for wear behavior and results showed that on increasing load, the wear rate and coefficient of friction also increased but on increasing sliding distance it decreased. While there was decrease in coefficient of friction when the sliding speed was increased before reaching the stable state. Graphite improved the wear resistance of composite due to its lubricating behavior. They used Taguchi method for finding the optimum process parameters. Roy et al. [4] found that wear rate of hybrid composite was decreased due to addition of its reinforcements. Also, the FSP lead to refinement of grains which ultimately added increasing the wear resistance of composite. Raj et al. [14] fabricated hybrid composite via stir casting technique using CNT and E Glass fibers as reinforcements in AA 7075 matrix. Wear Test of composite showed that wear rate decreased upto a value of content of CNT and E Glass and after that it starts increasing. It was evident that wear rate increased with increase in applied load and sliding velocity. Rajesh et al. [15] found that hybrid composite with alumina and silicon carbide had higher wear resistance than the base alloy AA 7075. SiC being the hard phase improved the wear resistance of the hybrid composite. Raghavendra et al. [16] processed hybrid composite with AA 7075 as matrix and Alumina and Silicon Carbide as reinforcement using stir casting technique. Microstructure of composite revealed the uniform distribution of particles. The wear test was performed on the pin on disc setup as shown in Fig.2.[16]. They found that on increasing load and sliding distance wear rate was also increased. Also, at high speed wear rate was found to be less as compared to at low speed of disc. The weight fraction also improved the wear resistance of composite.
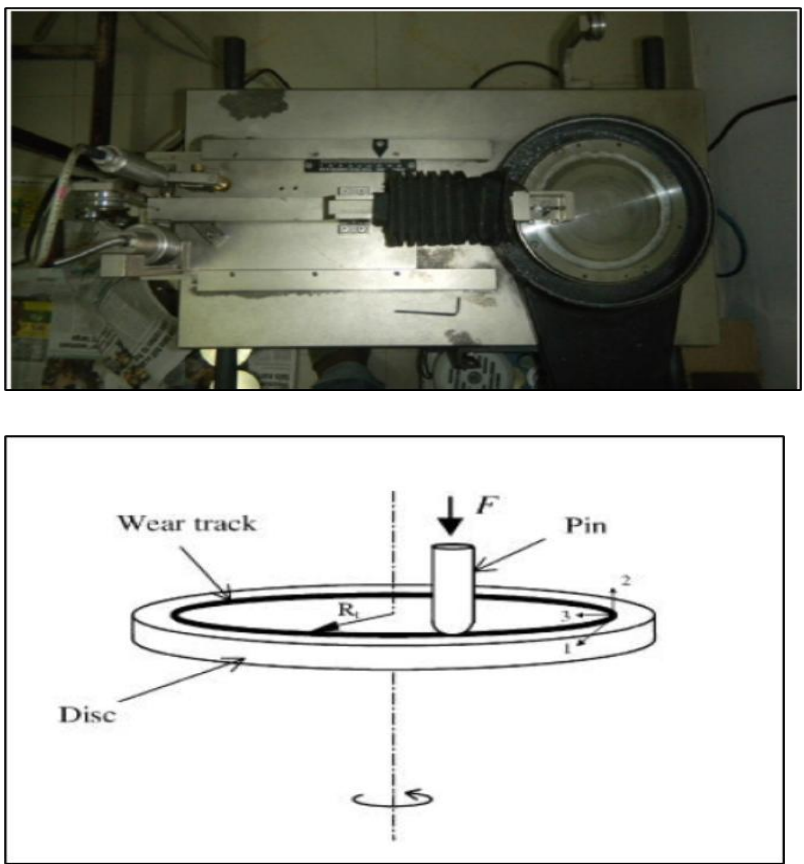

\section{Fig.2. Wear and Friction monitor and Pin on Disc geometry [16]}

Baradeswaran et al. [17] fabricated hybrid composite using alumina and graphite as reinforcements by stir casting method. Wear test results showed that graphite being good solid lubricant controlled the wear of composite by layer formation and by decreasing the coefficient of friction. Kumar et al. [18] for hybrid composite with Silicon Carbide and graphite as reinforcement studied the wear behavior. They found that for all combination of loads, sliding speed and sliding distance wear rate was less for hybrid composite than for the base alloy. They utilized RSM and found that the wear rate was mainly affected by the applied load.

\section{CORROSION BEHAVIOUR}

Ravikumar et al. [19] studied about the corrosion behavior of AA 7075 hybrid composite reinforced with alumina and silicon carbide. They found the corrosion rate and inhibitors efficiency of inhibition for the composite corrosion. Heat treatment of composite and increase in content of reinforcement resulted into increase in the efficiency of inhibitor. Consequently, there was decrement in corrosion rate and weight loss of specimens. The inhibitor was adsorbed continuously over the surface of hybrid composite and isolated it from the corrosive environment. Reinforcement was found to be efficient in inhibiting the corrosion of AA 7075 hybrid composite. Basavaraj et al. [20] prepared hybrid composite with CNT and E Glass fibers as reinforcement. Composite was then subjected to corrosion test in acidic medium and its behavior was analyzed. They found that exposure time played very important role while measuring corrosion rate. It is clearly evident that as the duration of test was increased, corrosion rate of composite decreased or corrosion resistance was increased.

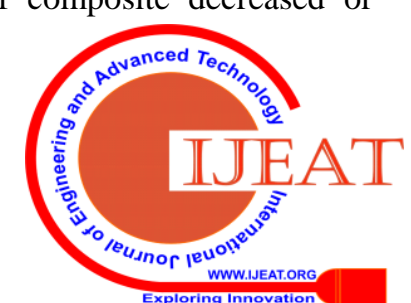




\section{Machining Characteristics, Tribological and Corrosion Behaviour of AA7075 Hybrid Composites}

This kind of relationship was mainly because of gradual development of protective layer over the surface of composite. They reported that as the content of e glass was increased the corrosion resistance also increased with it because of its hindrance to corrosion process and formation of intermetallic compounds in matrix. Hybrid composite were found to give more resistance to corrosion than the base alloy. They learned that as the normality was increased rate of corrosion was also increased. Sravanthi et al. [21] processed hybrid composite with AA 7075 as matrix, Silicon carbide and Graphite as reinforcements by liquid metallurgy technique. The corrosion behavior of composites was investigated by using weight loss method in which the samples are immersed in the solution of acid chloride, acid nitrate, acid sulphate and neutral chloride medium. Weight loss of the composite samples gives the rate of corrosion. They also reported that with increase in content of SiC the corrosion resistance of the composite was increased. While as the time span increased the corrosion rate of both the composite and alloy decreased. But on comparison among alloy and composite latter was found to be more corrosion resistant than former. It was also evident that corrosion rate was much less in neutral chloride medium as compared to all other mediums. The decreasing rate of corrosion with time can be attributed to the passivation of the composite in corrosive environment by formation of protective layer over the surface. Dixit [22] fabricated AA 7075 hybrid composite with $\mathrm{SiC}$ and Graphite as reinforcements. They studied about the corrosion behavior of composite under cryogenic condition and without it. It was observed that when there was no cryogenic condition as the content of $\mathrm{SiC}$ and Graphite was increased the number of pits and cracks also increased which indicated that there was decrease in corrosion resistance. While corrosion resistance increased with increase in reinforcement content under cryogenic condition it was mainly attributed to precipitation of microfine carbide over the surface of samples. Sambathkumar et al. [23] processed hybrid composite using two step stir casting technique with $\mathrm{SiC}$ and $\mathrm{TiC}$ as reinforcements. Then composite was subjected to potentiodynamic polarization testing. It was found that use of hard particles as reinforcements and its uniform distribution can enhance the corrosion resistance of composite. They reported that the corrosion resistance of 15 vol. \% of hybrid composite was greater than the base alloy in $3.5 \% \mathrm{NaCl}$ solution. Suresh et al. [24] investigated the corrosion behaviour of $\mathrm{Al} \mathrm{7075/} \mathrm{SiC}$ composite and $\mathrm{Al}$ 7075/nSiC/nAl2O3 hybrid composite reinforced with 1,2,3 $\& 4$ wt.\% of nano alumina and nano silicon carbide. They used weight loss method of corrosion testing and immersed the specimen for 96 hours in different corrosive media like 3.5\% sodium chloride, hydrochloric acid and sulphuric acid. They observed that the hybrid composite showed much higher corrosion resistance to single reinforced composite. Jaiswal et al [25] fabricated hybrid metal matrix composite by adding titanium carbide and aluminium oxide in aluminium alloy 7075. They investigated the mechanical and corrosion behaviour of hybrid composite and found that the corrosion resistance of composite got enhanced with concentration of aluminium oxide. George et al. [26] investigated for corrosion behaviour of chromium oxide and titanium boride reinforced
AA7075 hybrid composite. They reported that as content of chromium oxide increased the corrosion resistance also increased. Prema et al. [27] evaluated the corrosion behaviour of AA 7075, AA7075/B4C composite and AA7075/B4C/Al2O3 hybrid composite. They found that composites possessed the better corrosion resistance as compared to base alloy. They reported that increase in content of B4C enhanced the corrosion resistance. It was also seen that the corrosion rate lowered with increase in exposure time. Bihari et al. [28] employed potentiodynamic polarization, electrochemical impedance spectroscopy and weight loss method to study the corrosion behaviour of alumina and graphite reinforced AA7075 hybrid composite. They observed the that alumina as reinforcement enhanced the corrosion resistance of hybrid composite and as the concentration of alumina increased the corrosion resistance of hybrid composite also increased as shown in Fig.3 [28].

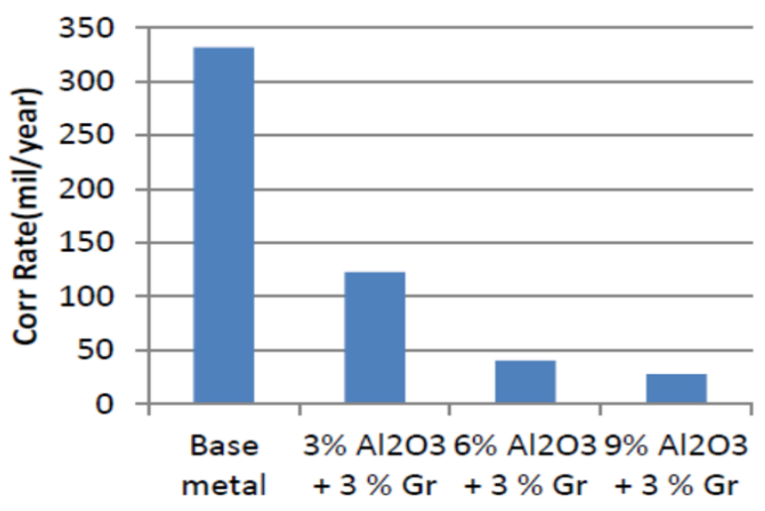

Fig.3. Corrosion rate for Base metal and different composition of Hybrid MMC [28]

Erek et al. [29] fabricated AA7075 hybrid composite reinforced with TiAl3 (in situ) and B4C (ex situ) using powder metallurgy method. They investigated the electrical conductivity and corrosion behaviour of the composite. They reported the deterioration in the corrosion resistance of the hybrid composite due to formation of interfaces between matrix and reinforcement phases. Kandasamy et al. [30] employed friction stir processing technique to enhance the corrosion resistance of AA7075/Al2O3/SiC hybrid composite. They performed potentiodynamic corrosion testing in $3.5 \% \mathrm{NaCl}$ solution. They reported that there was an improvement in the interface between matrix and reinforcement particle due to severe plastic deformation. This led to the enhancement of corrosion resistance of the composite. They also observed that as the higher content of $\mathrm{Al} 2 \mathrm{O} 3$ and lower content of SiC possessed the higher corrosion resistance than the other compositions.

\section{CONCLUSION}

In this article the machining characteristics, tribological and corrosion behaviour of AA 7075 hybrid composite were reviewed. In most of the cases it was found that hybrid composite was processed by using stir casting technique. Many of the researchers used friction stir processing

Retrieval Number: E9695069520/2020@BEIESP

DOI: 10.35940/ijeat.E9695.069520

Journal Website: www.ijeat.org
Blue Eyes Intelligence Engineering

\& Sciences Publication

(C) Copyriaht: All riahts reserved.

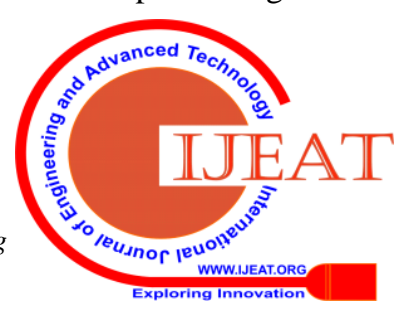


technique in order to achieve uniform dispersion of reinforcement particles in the matrix and ultra-fine equi axed grains. They were successful in achieving that at low tool travelling speed. Consequently, properties such as hardness and wear resistance were significantly improved after friction stir processing. Various investigations were carried out on machining of hybrid composite using Electric Discharge Machining technique. Further the optimization of process parameter such as current, voltage, pulse on time and pulse off time was done and their effect on material removal rate, surface roughness and power consumption were analysed by using RSM technique. Current was most influencing process parameter which affected the material removal rate utmost. Optimized process parameters were also found for Abrasive water jet machining technique. Investigation of tribological behaviour of hybrid composite revealed that selection of reinforcement is very much important in order to achieve better wear resistance. Various methods were also utilized for obtaining optimal combination of process parameters. Corrosion studies of hybrid composites reported that reinforcement played very important role in passivizing composite from corrosive media by forming a protective layer over it. Hybrid composites show better machining characteristics, tribological and corrosive behaviour than monolithic alloys and single reinforced composite. More such investigations need to be done for determining the other better and economic processes.

\section{REFERENCES}

1. R.Ramesh

Kumar,

K.A.Sudagar,G.Vijay,M.R.Raveendran,.

R,Shankar, Methodology and Enhancement technique for AA7075 Hybrid MMC

2. Mahanta S, Chandrasekaran M, Samanta S. EDM of Al7075-B4C-flyash hybrid metal matrix nano-composites and optimization of sustainable measures using genetic algorithm. ADBU Journal of Engineering Technology. 2017 Dec 31;6(3).

3. Kolli, Naresh, Tirumala Rao Chinka, Murahari kolli (2017). Experimental Studies on EDM of Al 7075/B4c/Gr Hybrid Composite using RSM Box-Behnken. International Journal for Research in Applied Science and Engineering Technology. V. 813-819. 10.22214/ijraset.2017.8114.

4. Pratip Roy, Subhash Singh \& Kaushik Pal (2017): Enhancement of mechanical and tribological properties of $\mathrm{SiC}$ - and CB-reinforced aluminium 7075 hybrid composites through friction stir processing, Advanced Composite Materials, DOI: 10.1080/09243046.2017.1405596

5. Naveen Kumar. K, Vinoth. R (2016): Analysis of Machining Characteristics of Al7075 Hybrid Metal Matrix Composite Using EDM, International Journal of Scientific Development and Research. Vol. 1, Issue 5.

6. Sasikumar, K.S.K., Arulshri, K.P., Ponappa, K. and Uthayakumar, M., 2018. A study on kerf characteristics of hybrid aluminium 7075 metal matrix composites machined using abrasive water jet machining technology. Proceedings of the Institution of Mechanical Engineers, Part B: Journal of Engineering Manufacture, 232(4), pp.690-704.

7. Kumar R, Chauhan S. Study on surface roughness measurement for turning of $\mathrm{Al}$ 7075/10/SiCp and $\mathrm{Al} 7075$ hybrid composites by using response surface methodology (RSM) and artificial neural networking (ANN). Measurement. 2015 Apr 1;65:166-80.

8. Lal S, Kumar S, Khan ZA, Siddiquee AN. Wire electrical discharge machining of AA7075/SiC/Al 2 O 3 hybrid composite fabricated by inert gas-assisted electromagnetic stir-casting process. Journal of the Brazilian Society of Mechanical Sciences and Engineering. 2014 Feb 1;36(2):335-46.

9. Yıldırım M, Özyürek D. An investigation of wear behaviors of AA7075 Al hybrid composites. High Temperature Materials and Processes. 2018 Jul 26;37(7):619-24.
10. Kumar PS, Reddy SP. Study of Hardness and Optimization of Wear Properties of AL 7075 Hybrid Composite.

11. Swathi B, Sriveni K, Harikrishna B. Study of Mechanical and wear properties of Al 7075/Al203p/Grp metal matrix composites. Indian J. Sci. Res. 2017;17(2):261-4.

12. Basithrahman A, Abirami S. Tribological Behaviour of AA 7075 Hybrid Composite using Stir Casting Method. International Journal of Engineering Research and Technology. 2017;6(1).

13. Imran M, Khan AA. Friction and Wear Properties of Al7075-BA-Gr Hybrid Composites.

14. Raj, Kiran, Dr. H. K. Shivanand and Dr. P. Vijayakumar. "Tribological Characterization of Al 7075 / CNT / E-Glass Reinforced Hybrid Particulate Metal Matrix Composite Developed by Stir Casting Process." (2016).

15. Rajesh AM, Kaleemulla M. Experimental investigations on mechanical and wear behavior of hybrid aluminium alloy. Volume. 2016 Sep;5:128-31.

16. Raghavendra N, Ramamurthy VS. Tribological Characterization of AL7075/AL203/SIC Reinforced Hybrid Particulate Metal Matrix Composite Developed by Stir Casting Process. International Journal of Recent advances in Mechanical Engineering (IJMECH). 2015 Aug;4.

17. Baradeswaran A, Perumal AE. Study on mechanical and wear properties of Al 7075/Al2O3/graphite hybrid composites. Composites Part B: Engineering. 2014 Jan 1;56:464-71.

18. Kumar R, Dhiman S. A study of sliding wear behaviors of Al-7075 alloy and Al-7075 hybrid composite by response surface methodology analysis. Materials \& Design. 2013 Sep 1;50:351-9.

19. Ravikumar M, Reddappa HN, Suresh R. Electrochemical studies of aluminium 7075 reinforced with $\mathrm{Al} 2 \mathrm{O} 3 / \mathrm{SiCp}$ hybrid composites in acid chloride medium. InAIP Conference Proceedings 2018 Apr 20 (Vol. 1943, No. 1, p. 020096). AIP Publishing LLC.

20. N. Basavaraj, Bylappa B. K., Dr. H. K. Shivanand*, Ravikumara S. Biradara, Nagabhushan V. Corrosion studies on cnt and e-glass fiber reinforced aluminium 7075 hybrid composites. World Journal of Engineering Research and Technology 2017, Vol. 3, Issue 5, 317-328.

21. Sravanthi M. , Manjunatha K. G. Corrosion Studies on Aluminium-7075 Alloy and its Composites by Weight Loss Method. International journal of innovative research \& development, Volume 5 , Issue 11, October 2016 (Special Issue).

22. Arun C Dixit U. Effect of cryogenic treatment on corrosion resistance of hybrid Aluminium7075 metal matrix composites. International Journal of Engineering Research and General Science Volume 4, Issue 3, May-June, 2016 ISSN 2091-2730.

23. Sambathkumar M, Navaneethakrishnan P, Ponappa KS, Sasikumar KS. Mechanical and corrosion behavior of Al7075 (hybrid) metal matrix composites by two step stir casting process. Latin american journal of solids and structures. 2017 Feb;14(2):243-55.

24. Suresh S, Gowd GH, Devakumar ML. Corrosion behaviour of $\mathrm{Al}$ 7075/Al 2 O 3/SiC MMNCs by weight loss method. Journal of Bio-and Tribo-Corrosion. 2018 Dec 1;4(4):62.

25. Jaiswal, S., Rajamurugan, G., Krishnasamy, P., Shaswat, Y. et al., "Mechanical and Corrosion Behaviour of Al 7075 Composite Reinforced with TiC and Al O Particles," SAE Technical Paper 2019-28-0094, 2019, https://doi.org/10.4271/2019-28-0094.

26. Lijin George, D S Robinson Smart and Vishnu Haridas, Evaluation of Tensile Strength and Corrosion Behaviour of Al 7075/ Tib2/ Cr2o3 Composite, International Journal of Mechanical Engineering and Technology, 10(2), 2019, pp. 59- 68.

27. Prema CE, Suresh S, Ramanan G, Sivaraj M. Characterization, corrosion and failure strength analysis of Al7075 influenced with B4C and Nano-Al2O3 composite using online acoustic emission. Materials Research Express. 2020 Jan 6;7(1):016524.

28. Bihari B, Prabhakar S, Singh AK. Corrosion Behaviour of $\mathrm{Al}$ 7075/Al2O3/Graphite Hybrid Composite in 3.5\% Sodium Chloride Solution. composites.;8:10.

29. Erek HB, Özyürek D, Asan A. Corrosion behaviour and electrical conductivity of reinforced $\mathrm{TiAl3}$ and B4C hybrid aluminium composites.

30. Kandasamy, Suganeswaran \& Rathinasamy, Parameshwaran \& N, Nithyavathy \& Arumugam, Karthik \& Rathanasamy, Rajasekar \& Kaliyannan, Gobinath. (2020). Corrosion behavioral studies on AA7075 surface hybrid composites tailored through friction stir processing. Anti-Corrosion Methods and Materials. ahead-of-print. 10.1108/ACMM-11-2019-2215.
Published By:

Blue Eyes Intelligence Engineering \& Sciences Publication

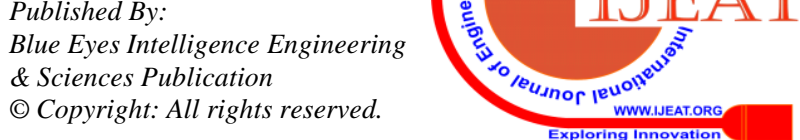


Machining Characteristics, Tribological and Corrosion Behaviour of AA7075 Hybrid Composites

\section{AUTHORS PROFILE}

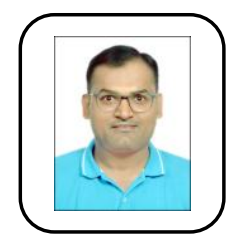

Abhishek Sharma, currently working as Ph.D. Scholar in the Department of Mechanical Engineering, Maulana Azad National Institute Technology, Bhopal, M.P. He received his M. Tech Degree in Stress \& Vibration Analysis in the Department of Mechanical Engineering from Maulana Azad National Institute Technology, Bhopal, M.P. He is currently working in the field of metal matrix composite and his research interest is in the field of material processing, characterization, heat treatment, corrosion and tribological behaviour.

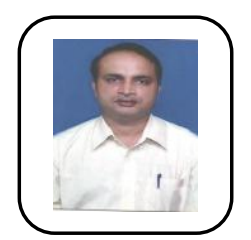

Dr.P.M. Mishra, currently working as Assistant Professor in the Department of Mechanical Engineering, Maulana Azad National Institute Technology, Bhopal, M.P. He completed his Ph.D. in the field of Facility planning plant in the year 2011. He has more than 50 research paper in the field of facility planning and metal matrix composite and having more than 15 year of research experience in the same field.

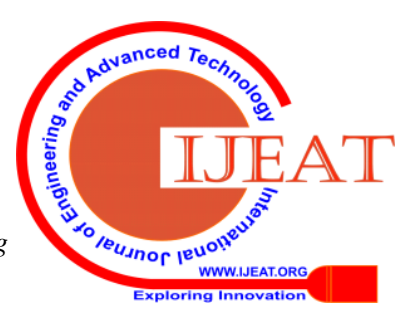

